\title{
Measuring the polarization response of a VNIR hyperspectral imager
}

\section{Authors: Riley D. Logan and Joseph A. Shaw}

Copyright None Society of Photo Optical Instrumentation Engineers. One print or electronic copy may be made for personal use only. Systematic reproduction and distribution, duplication of any material in this paper for a fee or for commercial purposes, or modification of the content of the paper are prohibited.

Logan, Riley D., and Joseph A. Shaw. "Measuring the Polarization Response of a VNIR Hyperspectral Imager." Edited by David B. Chenault and Dennis H. Goldstein. Polarization: Measurement, Analysis, and Remote Sensing XIV (April 21, 2020). doi:10.1117/12.2558257 


\title{
Measuring the polarization response of a VNIR hyperspectral imager
}

\author{
Riley D. Logan ${ }^{\mathrm{a}}$ and Joseph A. Shaw ${ }^{\mathrm{a}}$ \\ aElectrical and Computer Engineering Department, Montana State University, P.O. Box \\ 173780, Bozeman, USA
}

\begin{abstract}
As the applications of hyperspectral imaging rapidly diversify, the need for accurate radiometric calibration of these imaging systems is becoming increasingly important. When performing radiometric measurements, the polarization response of the imaging system can be of particular interest if the scene contains partially polarized objects. For example, when imaging a scene containing water, surface reflections from the water will be partially polarized, possibly affecting the response of the imaging system. In this paper, the polarization response of a Resonon, Inc. visible near-infrared (VNIR) hyperspectral imaging system is assessed across a spectral range of $400 \mathrm{~nm}$ to $1000 \mathrm{~nm}$, with a spectral resolution of $2.1 \mathrm{~nm}$. Efforts are currently underway to correct for the observed polarization response of the imaging system.
\end{abstract}

Keywords: Remote Sensing, Polarization, Radiometry

\section{INTRODUCTION}

Among the variety of remote sensing systems employed for gathering spectral and spatial information, hyperspectral imagers provide especially rich spectral information. Hyperspectral imaging systems are commonly used to gather detailed spectral information from scenes that may include partially polarized light, although they are generally partially sensitive to polarization and not designed for polarization imaging. ${ }^{1}$ In one such case, a hyperspectral imager was deployed with linear polarizers to assess the spectra of partially polarized surface reflections from oceanic waters as a function of polarizer angle. ${ }^{2,3}$ However, few studies have been completed to analyze the polarization response of hyperspectral imaging systems. ${ }^{4}$ This paper summarizes the results of polarization response measurements for the Resonon Pika L hyperspectral imager ${ }^{5}$ that operates over the visible and near infrared (VNIR) spectral range.

\section{BACKGROUND}

Polarization can be defined broadly as the vibrational state of the electric field of an electromagnetic wave. Typically, an electromagnetic wave generated from natural sources will take on a randomly polarized state. In other words, the electric field of the emitted light will continually fluctuate as a function of time, resulting in a net cancellation of any single polarization state.

Though randomly polarized light is common, the electric field components of an electromagnetic wave may take on many more orientations and phases, giving rise to numerous unique polarization states. For this paper, only linear polarization states will be discussed. That is, when the electric field vectors, $E_{x}$ and $E_{y}$, representing oscillations along the $\mathrm{x}$-axis and $\mathrm{y}$-axis, respectively, have no phase difference. With no phase difference, the electric field vectors will vibrate in the same plane. Depending on the values of $E_{x}$ and $E_{y}$, a variety of polarization states will be formed (Fig. 1). These linear polarization states will be exploited to determine the polarization response of the Pika L imager.

Further author information: (Send correspondence to Dr. Joseph A. Shaw)

Joseph A. Shaw: E-mail: joseph.shaw@montana.edu, Telephone: +1 406-994-7261 


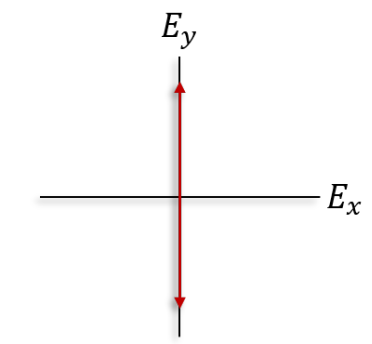

Vertical Polarization $\left(0^{\circ}\right)$

$E_{x}=0$

$E_{y}= \pm 1$

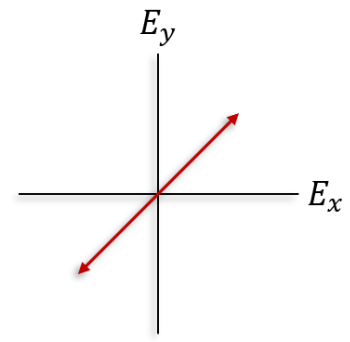

$45^{\circ}$ Polarization $\left(45^{\circ}\right)$

$E_{x}= \pm 0.5$

$E_{y}= \pm 0.5$

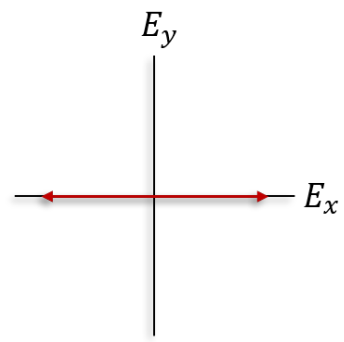

Horizontal Polarization $\left(90^{\circ}\right)$

$E_{x}= \pm 1$

$E_{y}=0$

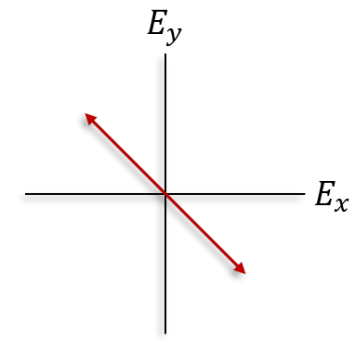

$135^{\circ}$ Polarization $\left(135^{\circ}\right)$

$E_{x}= \pm 0.5$

$E_{y}= \pm 0.5$

Figure 1. Linear polarization states with normalized values for $E_{x}$ and $E_{y}$. Polarizer angle shown in parentheticals.

\section{METHODS}

To create the variable linear polarization states used to determine the polarization response of the Pika L system, we used an integrating sphere and a large-aperture polarizer on a rotation stage. This produced a spatially uniform source of linearly polarized light with a user-selected orientation. The test polarizer was a 129-mmdiameter wire-grid polarizer with an extinction ratio of at least 1000 between $400 \mathrm{~nm}$ to $1000 \mathrm{~nm}$ (Meadowlark Optics VLM-129-UV-C). We directed the Pika L, attached to a tripod-mounted rotation stage, to view the polarized light transmitted through the wire-grid polarizer from the integrating sphere by sweeping it through a small angular range of approximately $2^{\circ}$ centered on normal incidence, resulting in a maximum angle of incidence of $\pm 1^{\circ}$ on the polarizer (Fig. 2). Using the Resonon SpectrononPro software package, we set the Pika L to capture 200 lines with a framerate of $40 \mathrm{~Hz}$, an integration time of $21 \mathrm{~ms}$, and a gain of 0 .

To avoid stray light from entering the system, we baffled the path between the polarizer and imager, then shrouded the entire experimental setup as an additional precaution. Beginning with a vertical orientation, we rotated the polarizer clockwise (when looking into the polarizer and integrating sphere) through $180^{\circ}$ in steps of $2^{\circ}$. At each step, we collected three hyperspectral data cubes and averaged to reduce noise. Using the averaged data cubes, we converted units from digital number to radiance to account for the wavelengthdependent response of the imaging system. This was done using a previous radiometric calibration derived by relating measured digital numbers to the variable integrating sphere radiance with no polarizer or other elements in the optical path. Once the data were converted into radiometric units, we calculated the polarization response spectrum of the Pika $L$ from the change in spectral radiance as a function of polarizer angle. To further quantify the polarization response of the Pika L, we calculated the apparent $S_{0}, S_{1}$, and $S_{2}$ Stokes parameters and the apparent degree of linear polarization (DoLP) that indicates the instrument's polarization-dependent relative spectral response function.

\section{RESULTS AND DISCUSSION}

The Pika L shows a clear dependence on the polarization state of incoming light, especially in the near-infrared spectral range beyond $700 \mathrm{~nm}$ wavelength. To visualize the polarization-dependent response of the imaging system, we plotted the spectra measured at 90 different polarizer angles, along with the fixed source output spectrum after passing through the test polarizer, as defined by the integrating sphere calibration certificate after multiplication by the polarizer's typical spectral transmittance curve (Fig. 3). Each colored line in the spread of measured spectral radiance represents a different polarization state. The red line depicts the theoretical radiance curve output by the integrating sphere, with its internal aperture $90 \%$ open, after passing through the test polarizer. We attribute the difference between the theoretical source spectral radiance and measured source spectral radiance to deviations from typical reported performance in our test polarizer, resulting in additional loss factors that extend beyond spectral transmittance effects. The loss factors could potentially be accounted 


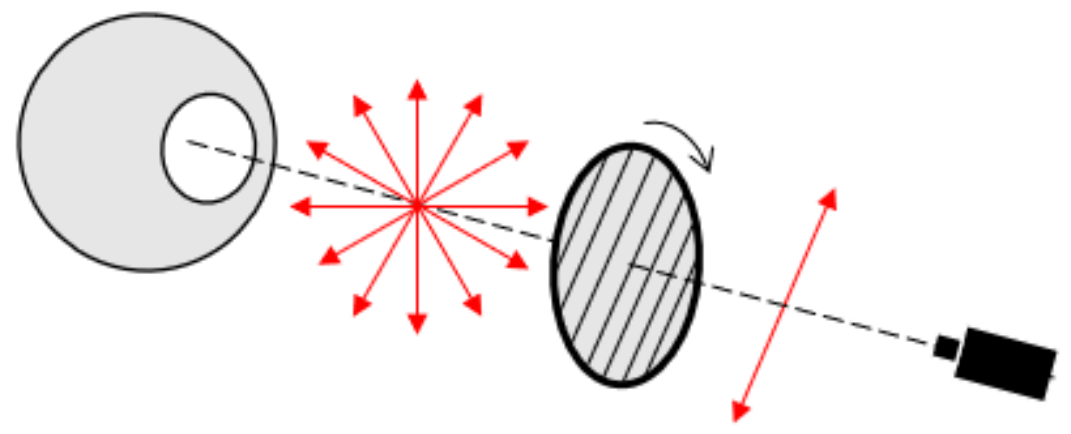

(a)

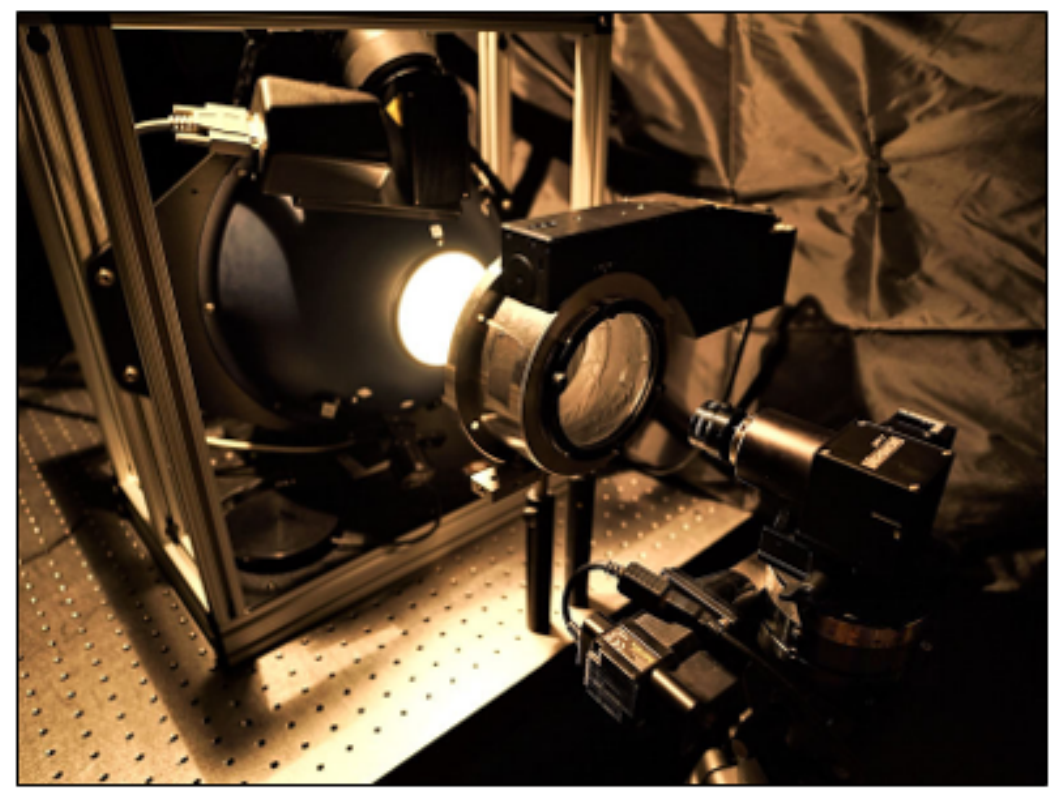

(b)

Figure 2. Experimental setup (a) and physical setup showing the integrating sphere, wire-grid linear polarizer, and Pika L imager without baffling in place (b).

for if a radiometric calibration were completed with the test polarizer in place, but this was not done for the measurements reported here.

Closer inspection of one wavelength with low polarization response $(614 \mathrm{~nm})$ and four wavelengths with strong polarization response $(682 \mathrm{~nm}, 744 \mathrm{~nm}, 828 \mathrm{~nm}, 958 \mathrm{~nm})$ helps to illuminate the dependence on polarizer angle (Fig. 4a-e). We plotted each of the selected wavelengths as a function of polarizer angle and normalized to the decimal fraction of the S0 Stokes parameter. If the Pika L had no polarization response, or we observed a wavelength with little polarization-dependence, the imager would be expected to record $S_{0} / 2$ across all polarizer angles, as given by Malus's Law (Fig. 4a). However, Figures 4b-e actually show a maximum variation of approximately $\pm 3.7 \%$ about $S_{0} / 2$. 


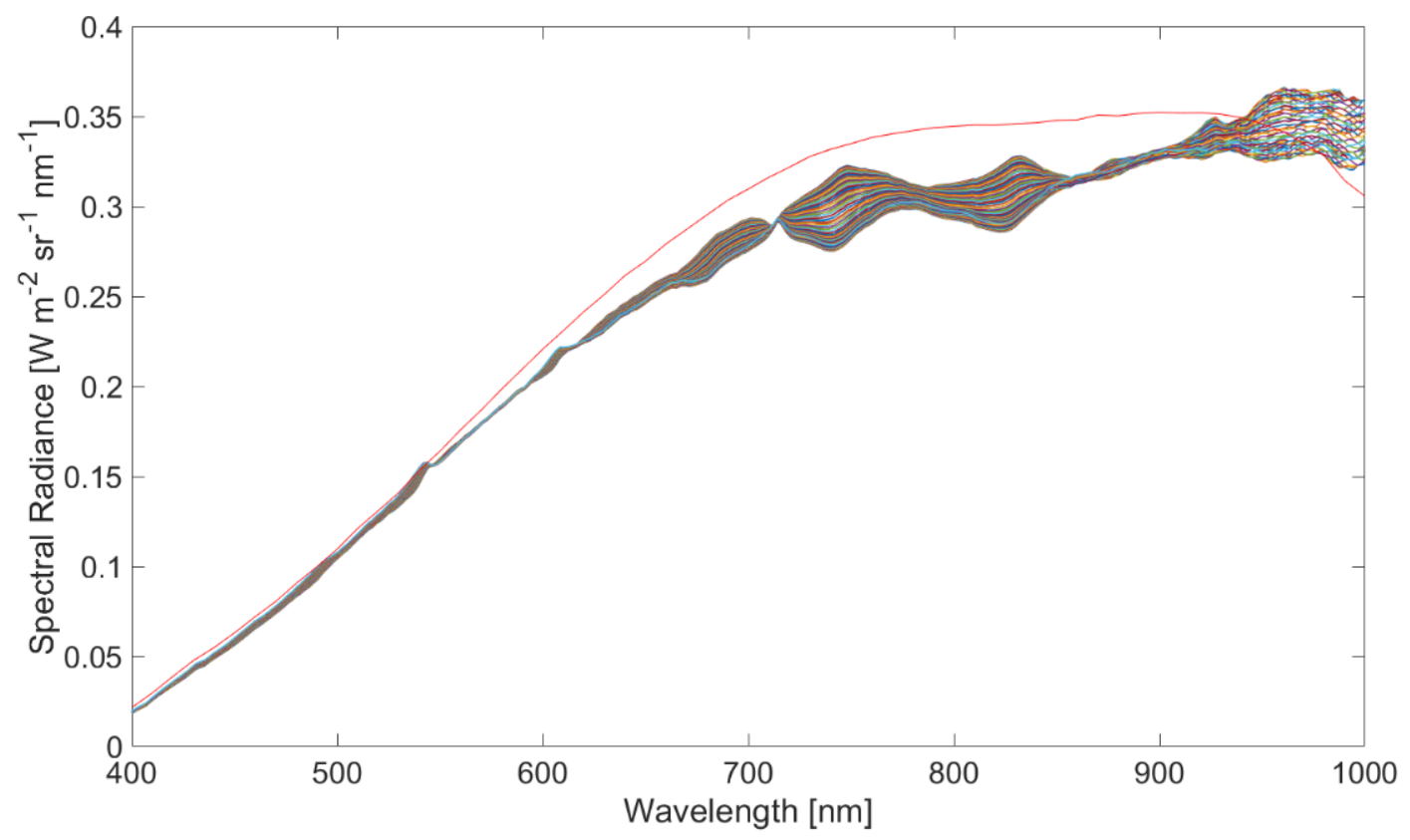

Figure 3. Integrating sphere output spectral radiance (red) and source spectral radiance measured by the Pika L at different polarizer angles (multi-color).

To better visualize the polarization response of the imager, we calculated the apparent degree of linear polarization (DoLP). To perform this calculation, we defined the $S_{0}, S_{1}$, and $S_{2}$ Stokes parameters as

$$
\begin{gathered}
S_{0}=L_{0^{\circ}}+L_{90^{\circ}} \\
S_{1}=L_{0^{\circ}}-L_{90^{\circ}} \\
S_{2}=L_{45^{\circ}}-L_{135^{\circ}},
\end{gathered}
$$

with $L_{0^{\circ}}, L_{90^{\circ}}, L_{45^{\circ}}$, and $L_{135^{\circ}}$ representing the detected spectral radiance for vertically, horizontally, $45^{\circ}$, and $135^{\circ}$ polarized light, respectively. Using these three Stokes parameters, we calculated the DoLP, which expresses the fraction of the light that is linearly polarized, as

$$
\operatorname{DoLP}=\frac{\sqrt{S_{1}^{2}+S_{2}^{2}}}{S_{0}} \text {. }
$$

Usually, the DoLP represents the decimal fraction of an incident electromagnetic wave that is linearly polarized, but here we are using the DoLP to quantify the amount of variation in the measurement of completely polarized light introduced by polarizer angle. In other words, we are using the DoLP to quantify the amount by which the measured signal extends above and below $S_{0} / 2$ as a function of polarizer angle for each wavelength. To visualize this quantity, we calculated the DoLP across all measured wavelengths for the Pika L imager (Fig. 5). As depicted in Figure 5, the Pika L imager has a polarization response that depends strongly on wavelength. The DoLP varies between a minimum value of approximately $0.001(0.1 \%)$ at $614 \mathrm{~nm}$ to a maximum value of $0.073(7.3 \%)$ at $744 \mathrm{~nm}$. 


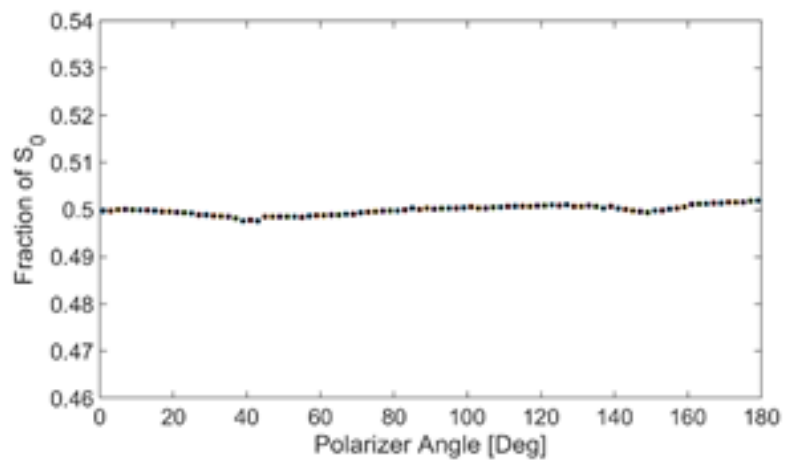

(a) $614 \mathrm{~nm}$

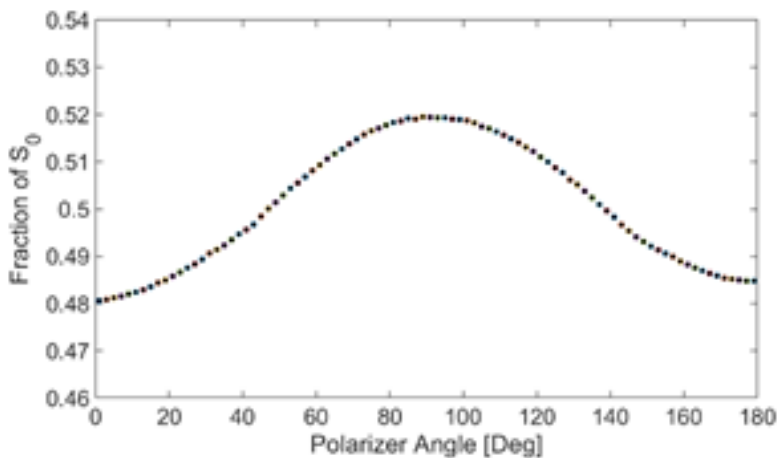

(b) $682 \mathrm{~nm}$

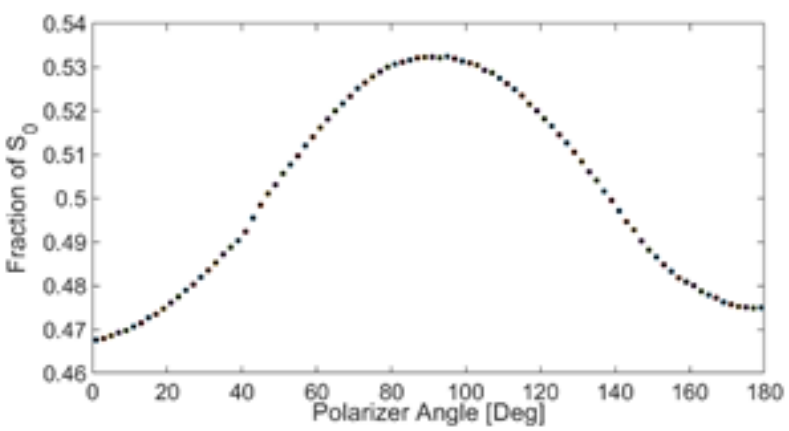

(d) $828 \mathrm{~nm}$

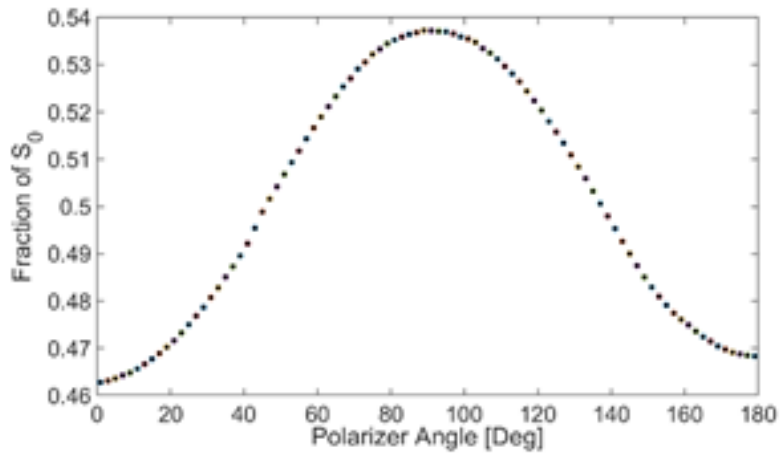

(c) $744 \mathrm{~nm}$

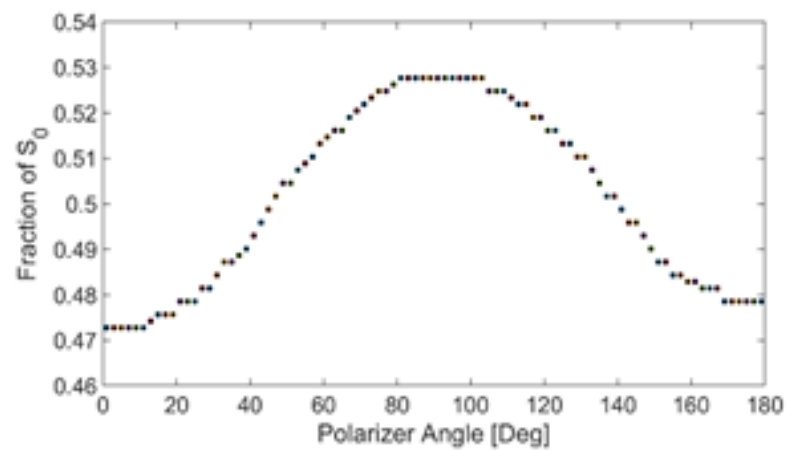

(e) $958 \mathrm{~nm}$

Figure 4. Polarization response of the Pika $\mathrm{L}$ in terms of $S_{0}$ as a function of polarizer angle at selected wavelengths. 


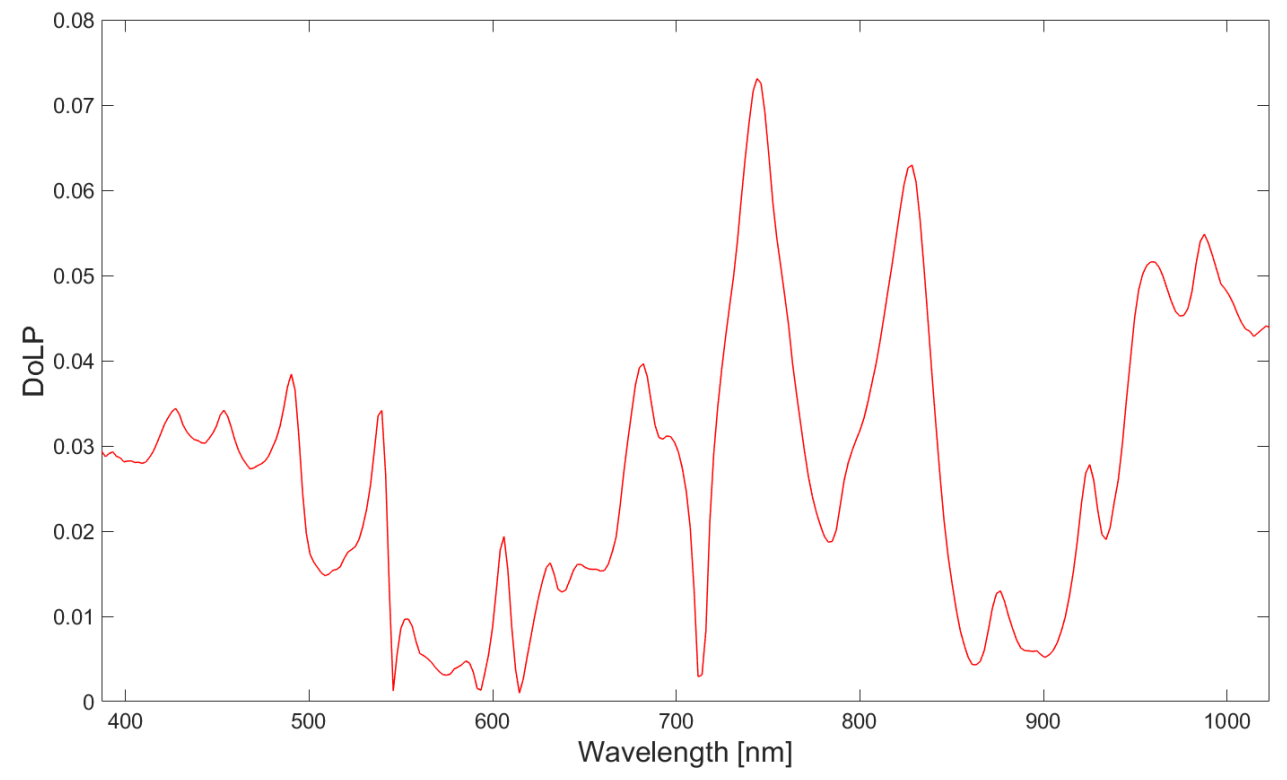

Figure 5. Degree of linear polarization as a function of wavelength for the Pika L imager.

\section{CONCLUSION}

By measuring its response to $100 \%$ linearly polarized light as a function of polarization angle and wavelength, we determined the polarization response of the Pika L hyperspectral imager. Uniform, randomly polarized light was sourced by an integrating sphere and linearly polarized by a wire-grid polarizer. The wire-grid polarizer was mounted in a precision rotation stage and rotated through $180^{\circ}$ in steps of $2^{\circ}$ to vary the polarization angle.

The results of this experiment show that the Pika L system has a polarization response which is strongly dependent on wavelength, with a minimum of $0.001(0.1 \%)$ at a wavelength of $614 \mathrm{~nm}$ and a maximum of 0.073 $(7.3 \%)$ at a wavelength of $744 \mathrm{~nm}$. Therefore, measurements with a desired uncertainty on the order of $10 \%$ could potentially be made without any concern for the polarization state of the light. Conversely, measurements with desired uncertainties of a few percent or less must carefully consider the polarization state of the incident light.

In future work, we plan to model and compensate the polarization response of the Pika L to retrieve accurate spectral radiance readings of polarized scenes. Correction of the observed polarization response would allow for more accurate imaging of polarized scenes and the ability to detect polarized objects in a scene.

\section{ACKNOWLEDGMENTS}

This material is based upon work supported in part by the National Science Foundation EPSCoR Cooperative Agreement OIA-1757351. Any opinions, findings, and conclusions or recommendations expressed in this material are those of the author(s) and do not necessarily reflect the views of the National Science Foundation.

\section{REFERENCES}

[1] Tyo, J. S., Goldstein, D. L., Chenault, D. B., and Shaw, J. A., "Review of passive imaging polarimetry for remote sensing applications," Applied Optics 45(22), 5453-5469 (2006).

[2] Gilerson, A., Carrizo, C., Foster, R., Harmel, T., Golovin, A., El-Habashi, A., Herrera, E., and Wright, T., "Total and polarized radiance from the ocean surface from hyperspectral polarimetric imaging," in [SPIE Defense + Commerical Sensing ], (11014), 96-106 (2019). 
[3] Gilerson, A., Carrizo, C., Ibrahim, A., Foster, R., Harmel, T., El-Habashi, A., Lee, Z., Yu, X., Ladner, S., and Ondrusek, M., "Hyperspectral polarimetric imaging of the water surface and retrieval of water optical parameters from multi-angular polarimetric data," Applied Optics 59(10), C8-C20 (2020).

[4] Aval, J., Alfalou, A., and Brosseau, C., "Polarization and hyperspectral imaging matter for newly emerging perspectives in optical image processing: guest editorial," Advances in Optics and Photonics 11(2), ED10-ED14 (2019).

[5] Resonon, “Hyperspectral Imaging Cameras And Systems," tech. rep., Resonon, Inc., Bozeman (2019). 